\title{
New Axion and Hidden Photon Constraints from a Solar Data Global Fit
}

\author{
Núria Vinyoles ${ }^{1}$, Aldo Serenelli ${ }^{1}$, Francesco Villante ${ }^{2}$, Sarbani Basu ${ }^{3}$, Javier Redondo ${ }^{4}$, Jordi \\ Isern $^{1}$ \\ ${ }^{1}$ Institute of Space Sciences (CSIC-IEEC), Campus UAB, 08193 Cerdanyola del Vallès, Spain \\ ${ }^{2}$ Dipartimento di Scienze Fisiche e Chimiche, Università dell'Aquila, I-67100 L'Aquila, Italy \\ ${ }^{3}$ Department of Astronomy, Yale University, PO Box 208101, New Haven, CT 06520, USA \\ ${ }^{4}$ Departamento de Física Teórica, Universidad de Zaragoza, 50009 Zaragoza, Spain
}

DOI: http://dx.doi.org/10.3204/DESY-PROC-2015-02/vinyoles_nuria

\begin{abstract}
We present a new statistical analysis that combines helioseismology and solar neutrino observations to place upper limits to the properties of non standard weakly interacting particles. We present two applications to test the method: the well studied case of axions and the more novel case of low mass hidden photons. For axions we obtain an upper limit at $3 \sigma$ for the axion-photon coupling constant of $g_{a \gamma}<4.1 \cdot 10^{-10} \mathrm{GeV}^{-1}$. For hidden photons we obtain the most restrictive upper limit available accross a wide range of masses for the product of the kinetic mixing and mass of $\chi m<1.8 \cdot 10^{-12} \mathrm{eV}$ at $3 \sigma$. Both cases improve the previous solar constraints based on the Standard Solar Models.
\end{abstract}

\section{Introduction}

Many studies have focused on using the Sun for setting limits on the properties of different types of exotic particles. The Sun is by far the best-known star. The solar structure, revealed by helioseismology and solar neutrinos, is well determined, and accurate solar models give us information about the past, present and the future of the Sun [1]. While in some cases (e.g. axions) the most restrictive limits are not inferred from solar studies, the Sun remains the most useful benchmark for testing and validating both stellar models and different statistical approaches to constrain particle properties. Also, it is important to keep in mind that CAST [2] and the forthcoming IAXO $[3,4]$ are experiments specifically designed to detect exotic particles directly from the Sun, so having predictions of upper limits for expected solar fluxes for exotic particles remains an important aspect to be considered.

Solar constraints on particle properties have been generally derived from applying limits to variations of either neutrino fluxes $[5,6]$ or the sound speed profile derived from helioseismology [5]. However, a systematic approach aimed at combining different sources of data accounting in detail for the observational and theoretical errors is badly missing in literature.

The goal of this work is to extend the general statistical approach presented in [7] to constrain properties of particles (e.g. mass, coupling constant) making the best possible use of all the available information of the Sun, both observational and theoretical. For this purpose, we use the helioseismic data combined with the neutrino fluxes in a statistical approach that includes the theoretical and observational uncertainties and takes into account possible tensions among 
New Axion And Hidden Photon Constraints from a Solar Data Global Fit

data and solar model input parameters. We then derive solar limits for the well-studied hadronic axions - to gauge the performance of our statistical approach- and for the more novel case of hidden photons for which the Sun sets the most restrictive limits on the kinetic mixing parameter for small hidden photon masses, $m \lesssim \mathrm{eV}$.

\section{Standard Solar Models}

In this work we use standard solar models (SSMs) as reference models. SSMs have been computed using GARSTEC [8] and are calibrated to match the present-day solar radius $\mathrm{R}_{\odot}=6.9598 \cdot 10^{10} \mathrm{~cm}$, luminosity $\mathrm{L}_{\odot}=3.8418 \cdot 10^{33} \mathrm{erg} \mathrm{s}^{-1}$ and surface metal-to-hydrogen ratio $(\mathrm{Z} / \mathrm{X})_{\odot}$. The choice of this last constraint is critical because it essentially determines the distribution of metals in the entire solar structure and it has been the subject of much discussion over recent years in the context of the solar abundance problem $[9,10,11,12]$.

To avoid that the results depend on the solar abundance problem we use an SSM that best reproduces the thermal stratification of the Sun and the solar neutrino data. This model is calculated following the method used in [7] that lets the solar composition free and adjusts the input parameters in SSMs within their experimental uncertainties (nuclear cross sections, microsocopic diffusion

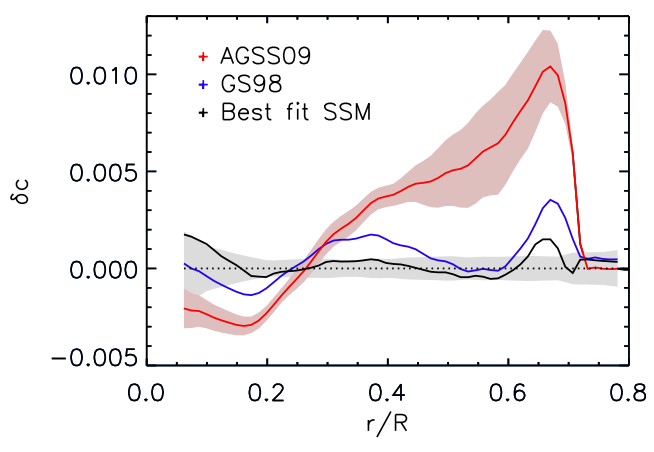

Figure 1: Comparison of sound speed profiles of SSMs. Red and blue lines are SSMs with AGSS09 and GS98 reference compositions and all input SSM parameters fixed to their central values. The black line shows results for the best SSM [7] resulting from finding the SSM with free composition. rate, etc.). In Fig. 1 we have plotted the SSM using different solar composition (GS98 [13] and AGSS09 [14]) and the best fit resulting from letting the composition free, showing that this last model matches the thermal stratification of the Sun, and thus, is a good model to be used as reference model.

\subsection{SSMs with axions and hidden photons}

We have calculated different SSM adding an extra energy-loss rate in the GARSTEC code resulting from the presence of axions or hidden photons. The dominant production of axions in the Sun comes from the Primakoff processes (conversion of a photon to an axions in presence of electro-magnetic fields) and the energy-loss rate used is the one in [5]. For axions, we aim to constrain the axion-photon coupling constant $\left(g_{a \gamma}\right)$. For hidden photons, we have only considered in this paper the longitudinal component. Hidden photons are produced by the conversion of a photon to a hidden photon, whose probability depends on the hidden photon mass $(m)$ and the kinetic mixing constant $(\chi)$. The product $\chi m$ is the parameter that can be constrained. The limits derived from the Sun will be valid for the mass range $m_{H P}<0.3 \mathrm{keV}$ because for the longitudinal hidden photons, the resonance emission will occur when its mass is equal or smaller than the plasma frequency of the $\operatorname{Sun}\left(\omega_{P}\right)$ as it is explained in [15]. The energy-loss rate used is taken from [15]. In Fig. 2 we show some of the results for SSM with 
axions and hidden photons before marginalizing over the composition in order to understand how the energy loss affects the structure and evolution of the Sun.
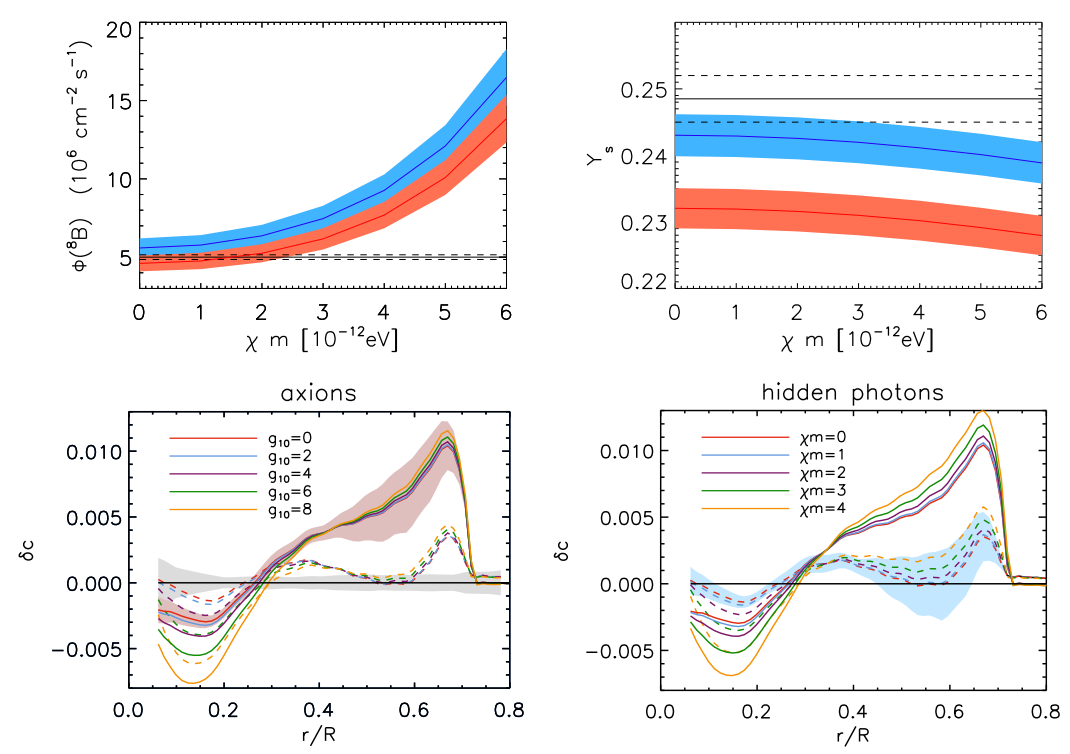

Figure 2: Upper panel: ${ }^{8} \mathrm{~B}$ and $Y_{s}$ as a function of $\chi m$ for hidden photons. Blue lines correspond to the GS98 composition and red ones to the red ones to AGSS09. Shaded lines show the model error and black lines the observational value and their errors. Lower pannel: Sound speed profile for hidden photons and axions for different values of $g_{10}$ and $\chi m$. Solid lines correspond to models with AGSS09 composition and dashed ones to GS98. Red and blue shaded zones correspond to the model errors and the grey one to the observational ones.

\section{Method and statistical procedure}

The statistical approach is based on the procedure presented in [7] that constructs a $\chi^{2}$ function that uses a figure-of-merit for the quality of different solar models in reproducing the observables. We build this function by considering 34 different observable quantities: the neutrino fluxes $\Phi\left({ }^{8} \mathrm{~B}\right)$ and $\Phi\left({ }^{7} \mathrm{Be}\right)$; the convective envelope properties $\mathrm{Y}_{\mathrm{S}}$ and $\mathrm{R}_{\mathrm{CZ}}$ and the sound speed determinations $\mathrm{c}_{i} \equiv c\left(r_{i}\right)$ for 30 different value of $r / \mathrm{R}_{\odot}$ where $r / \mathrm{R}_{\odot}<0.80$.

The bounds on axions and hidden photons are obtained by marginalizing with respect to the surface composition (best fit model), i.e. for each assumed value of $g_{10}$ and $\chi m$ we rescale the surface abundances of volatile and refractory elements by the factors $\left(1+\delta z_{\mathrm{vol}}\right)$ and $\left(1+\delta z_{\text {met }}\right)$ in order to achieve the best possible agreement with observational data (best fit model). Then, the results have a very minimal dependence on the reference solar composition used. For simplicity, we show here the results obtained by using the AGSS09 as reference composition (i.e. as pivot point for expansion in $\delta z_{\text {ref }}$ and $\delta z_{\mathrm{vol}}$ ). Identical results are obtained if GS98 composition is instead used. 


\section{New Axion and Hidden Photon Constraints from a Solar Data Global Fit}

\section{Results}

In Fig. 3, we show the $\chi^{2}$ function after marginalizing for the composition as function of $g_{10}$ and $\chi m$. This function has been calculated using: 1) all the observables combined, 2) only the sound speed profile, and 3) the neutrino fluxes combined with the surface helium and the convective radius. This way is useful to understand how much of each piece of experimental information contributes to the bounds. The sound speed profile gives the most restrictive limit, however, the neutrino fluxes and the convective parameters also have a noticeable contribution to the global bound. By setting a limit at $\Delta \chi^{2}=9$ we derive the upper bound $g_{10}<4.1$ at a 3- $\sigma$ CL for axions, almost a factor of 2 lower than previous solar limits, and the upper bound $\chi m=1.8 \cdot 10^{-12} \mathrm{eV}$ at a $3-\sigma \mathrm{CL}$.

For a longer discussion and more details on the method and the results, see [16].

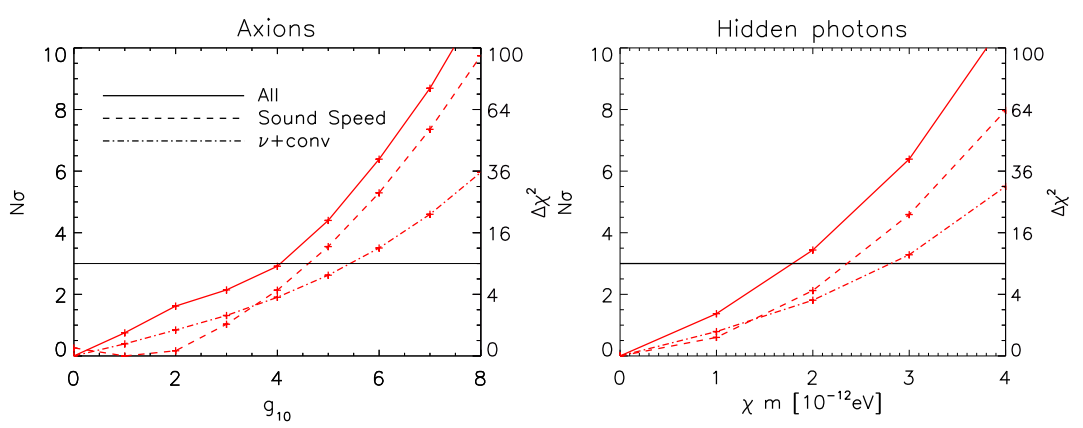

Figure 3: Values of $N \sigma$ and $\Delta \chi^{2}$ for models with axions and hidden photons. Solid line: using all observables $\Phi\left({ }^{7} \mathrm{Be}\right), \Phi\left({ }^{8} \mathrm{~B}\right), \mathrm{Y}_{\mathrm{s}}, \mathrm{R}_{\mathrm{CZ}}$ and 30 points of the sound speed profile. Dashed line: using the sound speed. Dotteddashed line: using the neutrinos and convective envelope properties.

\section{References}

[1] A.M. Serenelli, W.C. Haxton and C. Peña-Garay, ApJ 743, 24 (2011) [arXiv:1104.1639].

[2] K. Zioutas et al., Physics Research A 425, 480 (2010) [astro-ph/9801176].

[3] I. collaboration, IAXO - The International Axion Observatory, http://iaxo.web.cern.ch.

[4] IAXO Collaboration, E. Armengaud et al., JINST 9, 5002 (2014) [arXiv:1401.3233].

[5] H. Schlattl, A. Weiss, and G. Raffelt, Astropart. Phys. 10, 353 (1999) [hep-ph/98].

[6] P. Gondolo and G.G. Raffelt, Phys. Rev. D 79, 107301 (2009) [arXiv:0807.2926].

[7] F. L. Villante, A. M. Serenelli, F. Delahaye, and M. H. Pinsonneault, ApJ 787, 13 (2014) [arXiv:1312.3885].

[8] A. Weiss and H. Schattl, Ap\&SS 316, 99 (2008).

[9] S. Basu and H. M. Antia, ApJL 606, L85 (2004) [astro-ph/0403485].

[10] J. N. Bahcall, A. M. Serenelli, and S. Basu, ApJL 621, L85 (2005) [astro-ph/0412440].

[11] A. M. Serenelli, S. Basu, J. W. Ferguson, and M. Asplund, ApJL 705, L123 (2009) [arXiv:0909.2668].

[12] J. A. Guzik and K. Mussack, ApJ 713, 1108 (2010) [arXiv:1001.0648].

[13] N. Grevesse and A.J Sauval, Space Sci. Rev. 85, 161 (1998).

[14] M. Asplund, N.Grevesse, A.J. Sauval and P. Scott, ARA\&A 47, 481 (2009) [arXiv:0909.0948].

[15] J. Redondo and G. Raffelt, JCAP 8, 34 (2013) [arXiv:1305.2920].

[16] N. Vinyoles, A. Serenelli, F.L. Villante, S. Basu, J. Redondo and J. Isern, arXiv:1501.01639. 